\title{
Prognostic value of matrix Gla protein in breast cancer
}

\author{
KEIKO YOSHIMURA $^{1,6}$, KENGO TAKEUCHI $^{2}$, KOICHI NAGASAKI $^{1}$, \\ SOICHI OGISHIMA ${ }^{4}$, HIROSHI TANAKA ${ }^{4}$, TAKUJI IWASE ${ }^{3}$, FUTOSHI AKIYAMA ${ }^{2}$, \\ YOSHIKAZU KURODA ${ }^{6}$ and YOSHIO MIKI ${ }^{1,5}$
}

\author{
${ }^{1}$ Genome Center, Japanese Foundation for Cancer Research; Divisions of ${ }^{2}$ Pathology, and \\ ${ }^{3}$ Breast Oncology, Cancer Institute Hospital, Tokyo 135-8550; Departments of ${ }^{4}$ Bioinformatics, and \\ ${ }^{5}$ Molecular Genetics, Medical Research Institute, Tokyo Medical and Dental University, Tokyo 113-8519; \\ ${ }^{6}$ Department of Surgery, Graduate School of Medicine, Kobe University, Kobe, Hyogo 650-0017, Japan
}

Received December 4, 2008; Accepted February 24, 2009

DOI: $10.3892 / \mathrm{mmr} 00000135$

\begin{abstract}
To assess the prognostic value of matrix Gla protein (MGP) expression in cases of breast cancer, 9 samples from patients diagnosed with breast cancer who were followed up for more than 10 years were microdissected and then analyzed using Affymetrix U133 Plus 2.0 Arrays. Genes that exhibited significant differences in expression between patients with a good prognosis and those with a poor prognosis were identified. The MGP gene was among the genes up-regulated in cases where the prognosis was poor, indicating that the mRNA levels of MGP are a potential prognostic indicator of breast cancer. However, immunohistostaining of breast tissue microarrays $(n=207)$ did not reveal a correlation between the protein expression of MGP and overall survival, neither was there a correlation between the protein expression of MGP and ER status or bone metastasis. In breast cancer cases, the mRNA level of MGP may be a marker indicating poor prognosis; however, protein expression determined by immunohistostaining is not.
\end{abstract}

\section{Introduction}

Matrix Gla protein (MGP) is a calcium-binding protein isolated from bone matrix and cartilage that requires vitamin $\mathrm{K}$ dependent $\gamma$-carboxylation (1-3). In addition to its presence in bone or cartilage, MGP is distributed in organs such as the lung, heart, kidney and spleen (4). Mutations in the MGP gene are noted in Keutel Syndrome (5), a rare condition characterized by ectopic calcification and ossification, peripheral stenosis of the pulmonary artery and midfacial hypoplasia. It has been suggested that the function of MGP in bone and cartilage is to inhibit the formation of hydroxyapatite.

Correspondence to: Dr Yoshio Miki, Genome Center, Japanese Foundation for Cancer Research, 3-10-6, Ariake, Koto-ku, Tokyo 135-8550, Japan

E-mail: miki@jfcr.or.jp

Key words: breast cancer, matrix Gla protein, gene expression microarray, tissue microarray
The role of MGP in oncogenesis is unclear, and the correlation between MGP expression and cancer differs according to tumor type. MGP mRNA expression that was increased in comparison to normal tissue levels was found in ovarian cancer (6) and renal cell carcinoma (7). In renal cell carcinoma, a significant inverse correlation was observed between the level of MGP expression and tumor size, lymph node metastasis and tumor grade. In contrast, the expression of MGP mRNA was down-regulated in colorectal cancer cells compared to adjacent normal tissue, though this down-regulation was not correlated with histopathologic features, such as tumor progression, size and cell differentiation (8). MGP mRNA levels were significantly increased in high- compared to low-grade astrocytic gliomas (9).

In breast cancer, a study using cDNA hybridization revealed the expression of MGP to be 20-fold higher in the human metastatic breast cancer cell line 600PEI than in normal breast epithelium (10). In this study, we found MGP to be overexpressed in breast cancer patients with a poor prognosis compared to those with a good prognosis, on the basis of gene expression analysis using microarrays. We then used the tissue microarrays to clarify whether MGP protein levels determined by immunohistostaining could serve as a prognostic marker of breast cancer, and analyzed the correlation between MGP and ER status or bone metastasis.

\section{Materials and methods}

Breast cancer samples. Gene expression microarray samples were obtained from patients who underwent surgical resection between May 1995 and October 1997 at the Department of Breast Oncology of the Cancer Institute Hospital of the Japanese Foundation for Cancer Research (JFCR). Patients had tumors $>3 \mathrm{~cm}$ in diameter, and sufficient follow-up data spanning more than 10 years were available $(n=9)$. Tissue microarray (TMA) samples were obtained from patients who underwent surgical resection between October 1994 and December 1995 at the Department of Breast Oncology of the Cancer Institute Hospital of JFCR. Patients were selected for the study if the available paraffin block was large enough for the fabrication of TMA samples, and if sufficient follow-up data were available ( $n=207$; median follow-up time, 8.2 years). 
Table I. Clinical and histopathological characteristics of breast cancer patients involved in the microarray analysis.

\begin{tabular}{lcccccc}
\hline Patient no. & Tumor size $(\mathrm{cm})$ & LN & ER & PgR & Adjuvant treatment & Prognosis \\
\hline 1 & 6.0 & $1 / 23$ & - & - & CMF & Deceased \\
2 & 5.3 & $5 / 30$ & - & - & CMF & Deceased \\
3 & 5.2 & $16 / 28$ & + & - & CMF TAM & Deceased \\
4 & 5.2 & $4 / 19$ & - & - & Radiation & Deceased \\
5 & 5.0 & $0 / 20$ & - & - & CMF & Surviving \\
6 & 3.7 & $0 / 34$ & - & - & - & Surviving \\
7 & 6.0 & $0 / 50$ & - & - & - & Surviving \\
8 & 4.8 & $0 / 23$ & - & - & 5'-DFUR,EXE & Surviving \\
9 & 5.5 & $0 / 33$ & + & + & CMF & Surviving \\
\hline
\end{tabular}

LN, lymph node metastasis; ER, estrogen receptor; PgR, progesteron receptor; CMF, cyclophosphamide, doxorubicin and fluorouracil; 5'-DFUR, 5'-deoxy-5-fluorouridine; EXE, exemestane; TAM, tamoxifen.

Gene expression microarray analysis. Laser microbeam microdissection was used to collect pure populations of tumor cells in all samples, and total RNA extraction was carried out (RNeasy Micro Kit, Qiagen). With the total RNA (100 ng) of each sample, two-cycle cDNA synthesis and labeling of cRNA were carried out according to Affymetrix (Santa Clara, CA, USA) protocol (11). We used $20 \mu \mathrm{g}$ of biotin-labeled cRNA and broke down the full length to 35-200 base fragments. Then, $15 \mu \mathrm{g}$ of the broken cRNA was used to make a cocktail solution, which was placed in Gene Chip HG U133 plus 2.0 and hybridized for $16 \mathrm{~h}$ at $45^{\circ} \mathrm{C}$. The arrays were washed and stained using Fluidic station 450 (Affymetrix) and scanned using an Affymetrix GeneChip Scanner 3000. Expression values for each gene were calculated using Affymetrix GeneChip analysis software MAS 5.0.

The microarray data were analyzed using $\mathrm{R}$ software (http://www.r-project.org/). Expression data were normalized by the Robust Multichip Average (RMA) method (12) using Bioconductor and associated packages (Bioconductor 2.6.1, http://www.bioconductor.org). The data were then $\log _{2}$-transformed. Genes expressed differently between the poor and good prognosis groups were identified using the Significance Analysis of Microarrays (SAM) method with a fold change cutoff $>3.5$ (up-regulated in the poor prognosis group) or $<0.4$ (down-regulated); p-value <0.01 (13).

Real-time RT-PCR analysis. Expression of MGP was quantified by real-time RT-PCR. The templates and primer set were mixed with 2X QuantiTect SYBR-Green PCR Master Mix (Qiagen). $\beta$-actin was used as a control. Reactions were performed in triplicate in 96-well microtiter plates in an ABI PRISM 7900HT (Applied Biosystems, Foster City, CA, USA). Primer sequences for MGP were 5'-GCTCAATAGGGAAG CCTGTGAT-3' (forward primer) and 5'-TTTCTTCCCTCA GTCTCATTTGG-3' (reverse primer); primer sequences for B-actin were 5'-TCACCCACACTGTGCCCATCTACGA-3' and 5'-CAGCGGACCGCTCATTGCCAATGG-3'.

Tissue microarray. TMAs of 207 breast cancer cases with at least two cores of $2 \mathrm{~mm}$ in diameter were made by punching out of the donor block and transferring to a recipient block according to the manufacturer's instructions using a dedicated TMA instrument (KIN-1, Azumayaikakikai, Tokyo, Japan).

Immunohistochemistry. Expression of MGP in breast cancer cells was validated by immunohistochemistry. Immunohistochemical analysis was performed according to the dextranpolymer method (EnVision+; Dako, Glostup, Denmark) using monoclonal antibodies against MGP (Proteintech Group Inc., Chicago, IL, USA; 1:100). Heat-induced antigen retrieval pretreatments were performed with Target Retrieval Solution (Dako). Antibody binding was scored in a blinded fashion by two pathologists. A score of 1 was assigned when $\geq 5 \%$ of the neoplastic cells were definitely positive and a score of 0 when $<5 \%$ of tumor cells were stained (Fig. 1).

Statistical analysis. Survival curves were plotted according to the Kaplan-Meier method and compared using log-rank tests by Statistica 5.5. Univariate and multivariate analyses of prognostic indicators of overall survival were performed using the Cox proportional hazards regression model with $\mathrm{R}$ software. Variables that were statistically significant at $\mathrm{p}<0.05$ were retained in the model.

\section{Results}

Gene expression analysis. The characteristics of the patients included in the gene expression microarray are summarized in Table I. We selected genes that were differentially expressed between the good prognosis group (overall survival $>10$ years) and the poor prognosis group (overall survival $<10$ years), and obtained 18 up-regulated $(\mathrm{FC}>3.5 ; \mathrm{p}<0.01)$ and 23 downregulated $(\mathrm{FC}<0.4 ; \mathrm{p}<0.01)$ genes in the poor prognosis group (Table II). MGP was among the genes overexpressed in the poor prognosis group $(\mathrm{FC}=9.25, \mathrm{p}=0.0012)$, and had the lowest p-value for discriminating between the two groups. Moreoever, the differential expression of MGP was determined by two probe sets (ID 238481_at and 202291_s_at).

Confirmation of differential expression. To confirm the results of gene expression microarray analysis, we carried out 


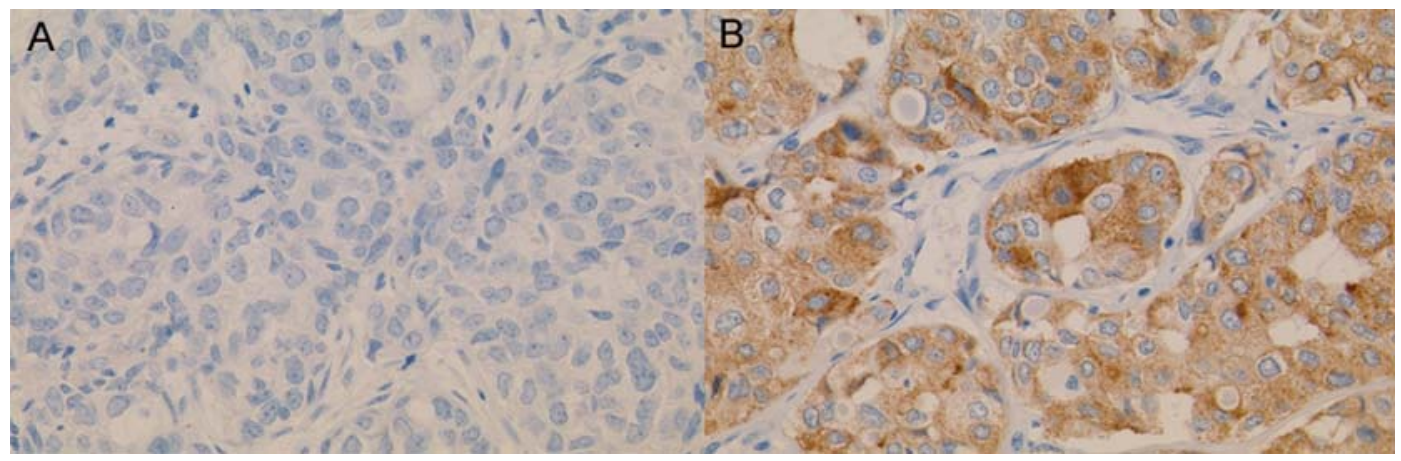

Figure 1. Representative images of the immunohistochemical staining of MGP in breast cancer tissue on a tissue microarray. (A) A score of 0 was assigned when $<5 \%$ of tumor cells were stained. (B) A score of 1 was assigned when at least $5 \%$ of the neoplastic cells were definitely positive. Original magnification x40 (objective).

A

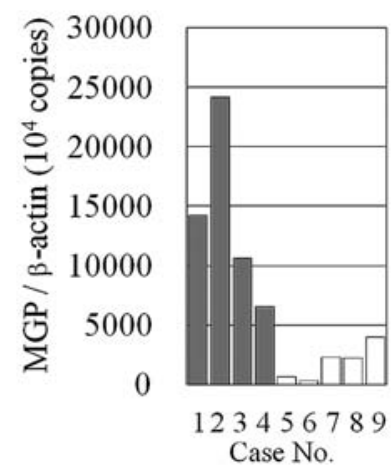

B

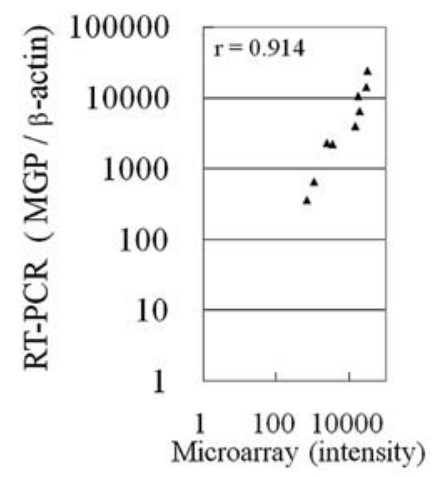

Figure 2. Gene expression microarray data were validated by quantitative RT-PCR. (A) Quantitative RT-PCR data showing upregulated expression of MGP in the poor prognosis group (gray bar). (B) Pearson correlation for MGP gene expression between microarray data and RT-PCR data, 0.914.

real-time quantitative RT-PCR for the MGP gene and the quantitative control gene, $\beta$-actin. Expression levels quantified by real-time RT-PCR were highly correlated with those determined by gene expression microarray analysis (Pearson rank correlation; $r=0.914)$, confirming the reliability of our microarray experiments (Fig. 2). B-actin was used as a reference gene, because it was the gene with the least fluctuation in our samples according to the microarray data. Expression of MGP was significantly higher in the poor than in the good prognosis group according to RT-PCR data $(\mathrm{p}<0.01)$. MGP mRNA levels were increased $>3$-fold in all the patients in the poor prognosis group, as compared to the mean expression level of the good prognosis group. The highest up-regulation of MGP observed was a 12-fold increase, as shown in Fig. 2. Based on the above findings, MGP was selected for further analysis.

Immunohistochemical analysis. MGP expression was examined in 207 breast cancer specimens by immunohistochemistry. The clinical and histopathological characteristics are listed in Table III. In the immunohistochemical evaluation, 104 patients had a score of 0 and 103 a score of 1 . We tested for associations between the stainings and overall survival. KaplanMeier overall survival curves are shown in Fig. 3. A score of 1 was not associated with a poor prognosis compared to a score of 0 (log rank, $\mathrm{p}=0.79)$.

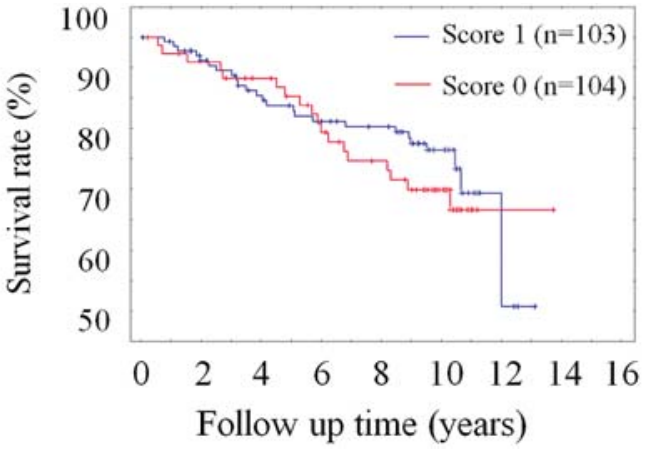

Figure 3. Kaplan-Meier curves of overall survival for MGP-negative (score 0) and MGP-positive (score 1) breast cancer cases. MGP protein expression was not significantly associated with overall survival $(\log$-rank, $p=0.79)$.

Regarding the correlation between MGP expression and ER status, the ER-positive rate was $60.6 \%$ for a score of 0 and $60.2 \%$ for a score of 1 . MGP protein expression was not related to ER status ( $\chi^{2}$ test, $\mathrm{p}=0.34$ ). The bone metastasis rate was $17.3 \%$ for a score of 0 and $12.6 \%$ for a score of 1 . MGP protein expression was not related to bone metastasis $\left(\chi^{2}\right.$ test, $\left.\mathrm{p}=0.96\right)$.

\section{Discussion}

Gene expression analysis revealed the mRNA level of MGP to be much higher in breast cancer patients with a poor prognosis than in those with a good prognosis. To investigate the utility of MGP protein expression as a prognostic marker of breast cancer, we examined the correlation between MGP protein expression and overall survival using tissue microarrays. First, the percentage of stained tumor cells, $-5,-25,-50,-75$, or $100 \%$, was determined, then the correlation between each of these groups and overall survival was analyzed. No difference between the groups was observed, and the correlation between MGP and overall survival was not confirmed.

A correlation between MGP expression and tumor development or tumor progression has been reported in ovarian cancer, renal cell carcinoma, colorectal cancer and glioma (6-9). There have also been several reports of the upregulation of MGP mRNA expression in human breast cancer cell lines $(10,14)$. 
Table II. Differentially expressed genes in the good and poor prognosis groups.

\begin{tabular}{|c|c|c|c|c|}
\hline Probe set & Gene symbol & Accession no. & P-value & Fold change \\
\hline \multicolumn{5}{|c|}{ Up-regulated genes in the poor prognosis group $(\mathrm{FC}>3.5, \mathrm{p}<0.01)$. } \\
\hline 227850_x_at & CDC42EP5 & AW084544 & 0.0076 & 9.78 \\
\hline 238481_at & MGP & AW512787 & 0.0012 & 9.26 \\
\hline 202291_s_at & & NM_000900 & 0.0072 & 3.81 \\
\hline 202859_x_at & IL8 & NM_000584 & 0.0062 & 8.73 \\
\hline 205476_at & CCL20 & NM_004591 & 0.0020 & 7.38 \\
\hline 37892_at & COL11A1 & $\mathrm{J} 04177$ & 0.0065 & 7.26 \\
\hline 203875_at & SMARCA1 & NM_003069 & 0.0047 & 5.57 \\
\hline 219359_at & ATHL1 & NM_025092 & 0.0039 & 4.76 \\
\hline 210306_at & L3MBTL & U89358 & 0.0087 & 4.75 \\
\hline 1561042_at & ITGB1 & AF086249 & 0.0076 & 4.64 \\
\hline 228877_at & RGL3 & AI379517 & 0.0028 & 4.31 \\
\hline 224646_x_at & H19 & BF569051 & 0.0048 & 4.07 \\
\hline 241937_s_at & WDR4 & AA577678 & 0.0065 & 3.94 \\
\hline 223380_s_at & LATS2 & AF207547 & 0.0017 & 3.79 \\
\hline 206595_at & CST6 & NM_001323 & 0.0014 & 3.64 \\
\hline 223251_s_at & ANKRD10 & $\mathrm{BC} 001727$ & 0.0061 & 3.64 \\
\hline 230136_at & LOC400099 & AI573252 & 0.0033 & 3.60 \\
\hline 219543_at & PBLD & NM_022129 & 0.0028 & 3.54 \\
\hline 238785_at & C3orf63 & AI632091 & 0.0053 & 3.53 \\
\hline \multicolumn{5}{|c|}{ Down-regulated genes in the poor prognosis group $(\mathrm{FC}<0.4, \mathrm{p}<0.01)$. } \\
\hline 223604_at & GARNL3 & AL136573 & 0.0014 & 0.39 \\
\hline 204560_at & FKBP5 & NM_004117 & 0.0014 & 0.38 \\
\hline 223824_at & C10orf59 & ВC005364 & 0.0088 & 0.37 \\
\hline 209823_x_at & HLA-DQB1 & M17955 & 0.0099 & 0.36 \\
\hline 205073_at & CYP2J2 & NM_000775 & 0.0097 & 0.35 \\
\hline 229332_at & HPDL & AI653050 & 0.0072 & 0.35 \\
\hline 205818_at & DBC1 & NM_014618 & 0.0073 & 0.31 \\
\hline 219389_at & SUSD4 & NM_017982 & 0.0063 & 0.31 \\
\hline 228160_at & LOC400642 & AI433706 & 0.0093 & 0.29 \\
\hline 209728_at & HLA-DRB4 & BC005312 & 0.0023 & 0.29 \\
\hline 211685_s_at & NCALD & AF251061 & 0.0020 & 0.28 \\
\hline 222774_s_at & NETO2 & AI335263 & 0.0025 & $0.28,0.19$ \\
\hline 218888_s_at & & NM_018092 & 0.0048 & \\
\hline 202741_at & PRKACB & AA130247 & 0.0096 & 0.26 \\
\hline 203290_at & HLA-DQA1 & NM_002122 & 0.0094 & 0.26 \\
\hline 209539_at & ARHGEF6 & D25304 & 0.0022 & 0.26 \\
\hline 223467_at & RASD1 & AF069506 & 0.0057 & 0.23 \\
\hline 206254_at & EGF & NM_001963 & 0.0017 & 0.23 \\
\hline 239911_at & ONECUT2 & $\mathrm{H} 49805$ & 0.0020 & $0.22,0.15$ \\
\hline 233446_at & & AU145336 & 0.0037 & \\
\hline 209315_at & HBS1L & AW297143 & 0.0058 & 0.18 \\
\hline 201943_s_at & CPD & NM_001304 & 0.0088 & 0.15 \\
\hline 205267_at & POU2AF1 & NM_006235 & 0.0088 & 0.14 \\
\hline 205029_s_at & FABP7 & NM_001446 & 0.0096 & $0.14,0.035$ \\
\hline 205030_at & & NM_001446 & 0.0057 & \\
\hline 225491_at & SLC1A2 & AL157452 & 0.0063 & 0.12 \\
\hline
\end{tabular}


Table III. Clinical and histopathological characteristics of the breast cancer patients.

No. of patients $(n=207)$

\section{MGP}

Score 0

104

Score 1

Age, years

$<40$

40-49

74

50-59

67

60-69

35

$\geq 70$

Lymph node metastasis

Negative

96

Positive

Tumor size (mm)

$\leq 19$

20-29

61

30-39

47

40-49

$50-59$

$\geq 60$

Estrogen receptor

Negative

Positive

Progesterone receptor

Negative

Positive

Her2

Negative

Positive

Adjuvant therapy

None

Done

Bone metastasis

Negative

Positive

In this study, using both microarray analysis and quantitative RT-PCR, we found that the mRNA of MGP was overexpressed in patients with a poor prognosis. This indicated that the mRNA levels of MGP could be a prognostic factor for breast cancer. However, we could not establish a correlation between the protein levels of MGP and overall survival. The reason for the difference in results regarding the mRNA and protein levels of MGP is unclear, though further study of the function of MGP may elucidate these findings. Estrogen has been found to strongly induce MGP gene expression in ERpositive breast cancer cells (14). However, in the present study, MGP expression was not significantly affected by ER status.

In summary, MGP mRNA expression was up-regulated in breast cancer patients with a poor prognosis, and may have the potential to serve as a prognostic indicator of the disease; however, no correlation was established between the protein levels of MGP as determined by immunohistostaining and overall survival.

\section{Acknowledgements}

This study was supported by a Grant-in-Aid for Scientific Research from the Ministry of Education, Culture, Sports, Science and Technology of Japan to Y.M. K.T. is a consultant for Dako. We thank all of the patients for their participation, and also thank Tomoyo Kakita, Ayumi Miura and Motoyoshi Iwakoshi for the technical assistance.

\section{References}

1. Price PA, Urist MR and Otawara Y: Matrix Gla protein, a new gamma-carboxyglutamic acid-containing protein which is associated with the organic matrix of bone. Biochem Biophys Res Commun 117: 765-771, 1983.

2. Price PA and Williamson MK: Primary structure of bovine matrix Gla protein, a new vitamin $\mathrm{K}$-dependent bone protein. J Biol Chem 260: 14971-14975, 1985.

3. Hale JE, Fraser JD and Price PA: The identification of matrix Gla protein in cartilage. J Biol Chem 263: 5820-5824, 1988.

4. Fraser JD and Price PA: Lung, heart, and kidney express high levels of mRNA for the vitamin K-dependent matrix Gla protein. Implications for the possible functions of matrix Gla protein and for the tissue distribution of the gamma-carboxylase. J Biol Chem 263: 11033-11036, 1988.

5. Keutel J, Jorgensen G and Gabriel P: A new autosomal-recessive hereditary syndrome. Multiple peripheral pulmonary stenosis, brachytelephalangia, inner-ear deafness, ossification or calcification of cartilages. Dtsch Med Wochenschr 96: 1676-1681 passim, 1971.

6. Hough CD, Cho KR, Zonderman AB, Schwartz DR and Morin PJ: Coordinately up-regulated genes in ovarian cancer. Cancer Res 61: 3869-3876, 2001.

7. Levedakou EN, Strohmeyer TG, Effert PJ and Liu ET: Expression of the matrix Gla protein in urogenital malignancies. Int $\mathrm{J}$ Cancer 52: 534-537, 1992.

8. Fan C, Sheu D, Fan H, Hsu K, Allen Chang C and Chan E: Down-regulation of matrix Gla protein messenger RNA in human colorectal adenocarcinomas. Cancer Lett 165: 63-69, 2001.

9. Van den Boom J, Wolter M, Kuick R, Misek DE, Youkilis AS, Wechsler DS, Sommer C, Reifenberger G and Hanash SM: Characterization of gene expression profiles associated with glioma progression using oligonucleotide-based microarray analysis and real-time reverse transcription-polymerase chain reaction. Am J Pathol 163: 1033-1043, 2003.

10. Chen L, O'Bryan JP, Smith HS and Liu E: Overexpression of matrix Gla protein mRNA in malignant human breast cells: isolation by differential cDNA hybridization. Oncogene 5: 1391-1395, 1990.

11. Technical note: Gene Chip Eukaryotic Small Sample Target Labeling Assay Version II. Available from URL: http://genomics. msu.edu/RTSF/small_sample_labeling.pdf.

12. Irizarry RA, Bolstad BM, Collin F, Cope LM, Hobbs B and Speed TP: Summaries of Affymetrix GeneChip probe level data. Nucleic Acids Res 31: e15, 2003.

13. Tusher VG, Tibshirani R and Chu G: Significance analysis of microarrays applied to the ionizing radiation response. Proc Natl Acad Sci USA 98: 5116-5121, 2001.

14. Sheikh MS, Shao ZM, Chen JC and Fontana JA: Differential regulation of matrix Gla protein (MGP) gene expression by retinoic acid and estrogen in human breast carcinoma cells. Mol Cell Endocrinol 92: 153-160, 1993. 